\title{
Human leishmaniasis in Brazil: A general review
}

\author{
Laís Anversa ${ }^{1 *} \odot$, Monique Gomes Salles Tiburcio², Virgínia Bodelão Richini-Pereira ${ }^{1}$, Luis Eduardo Ramirez ${ }^{3}$ \\ ${ }^{1}$ Scientific Researcher. Biomedical Sciences Core - Instituto Adolfo Lutz, Centro de Laboratório Regional de Bauru (IAL - CLR Bauru), Bauru, SP, Brazil \\ ${ }^{2}$ Adjunct Professor. Department of Health Sciences, Universidade Federal de Lavras (UFLA), Lavras, MG, Brazil \\ ${ }^{3}$ Full Professor. Department of Immunology, Microbiology and Parasitology, Instituto de Ciências Biológicas e Naturais, Universidade Federal do Triângulo Mineiro, Uberaba, MG, Brazi
}

\section{SUMMARY}

Study conducted at Instituto Adolfo Lutz

- CLR Bauru, Bauru, SP, Brazil

Article received: $7 / 17 / 2017$ Accepted for publication: 8/6/2017

*Correspondence: Instituto Adolfo Lutz - CLR Bauru

Leishmaniasis is a disease with ample clinical spectrum and epidemiological diversity and is considered a major public health problem. This article presents an overview of the transmission cycles, host-parasite interactions, clinical, histological and immunological aspects, diagnosis and treatment of various forms of the human disease.
\end{abstract} Address: Rua Rubens Arruda, Quadra 6, Centro Bauru, SP - Brasil Postal code: 17015-110 laisanversa@yahoo.com.br lanversa@ial.sp.gov.br
Keywords: Leishmaniasis. Protozoan Infections. Review.

\section{INTRODUCTION}

Leishmaniasis is caused by several species of digenetic protozoa of the order Kinetoplastida, family Trypanosomatidae and genus Leishmania, which affect humans and many animals. ${ }^{1}$ This parasite is endemic in at least 98 countries and approximately 0.2-0.4 and 0.7-1.2 million new cases of visceral leishmaniasis (VL) and cutaneous leishmaniasis (CL) occur every year, respectively. ${ }^{2}$

More than $90 \%$ of worldwide cases of VL occur in six countries (India, Bangladesh, Sudan, South Sudan, Ethiopia and Brazil) and despite a wide distribution, around one third of CL cases occur in the Americas, the Mediterranean basin and western Asia, mainly in Afghanistan, Algeria, Colombia, Brazil, Iran, Syria, Ethiopia, North Sudan, Costa Rica and Peru. Mortality data is extremely scarce and generally only represents hospital deaths. However, using an overall mortality rate of $10 \%$, we can conclude there are an estimated 20,000 to 40,000 annual deaths resulting from this parasitic disease. ${ }^{2}$

Due to the significant overall increase in its incidence, there has been growing interest in leishmaniasis in recent decades. In addition to reporting recent epidemics in endemic areas, there is evidence of dissemination of leishmaniasis to previously non-endemic areas. Such increases can be explained in part by improved diagnosis and reporting of cases, but they also result from factors associated with increased population migration, increased detection of leishmaniasis associated with opportunistic infections, the emergence of resistance to drugs used in treatment, and the adaptation of the transmission cycles to peridomiciliary environments due to urbanization and deforestation. ${ }^{3}$

In Brazil, autochthonous cases of CL have already been reported in all states and cases of VL have been recorded in 21 of the states, with approximately 1,600 cities showing autochthonous transmission. ${ }^{4,5}$

\section{DeVelopment}

Transmission

The transmission cycles of leishmaniasis vary according to geographic region, involving a wide diversity of species of Leishmania, vectors (invertebrate hosts) and reservoirs (vertebrate hosts). More than 50 species of Leishmania have been identified worldwide, and at least 21 of these species have significant medical importance. ${ }^{6}$ Particularly in Brazil, eight species have already been isolated from patients with the disease (Table 1). ${ }^{3,4}$

Vertebrate hosts of the various species of Leishmania include a wide variety of mammals such as rodents, canines, marsupials, edentates, carnivores, primates and, among these, humans. ${ }^{3}$ The vectors are the females of the insects called phlebotomine sand flies belonging to the order Diptera, family Psychodidae, subfamily Phlebotominae and genus Phlebotomus, in the Old World, and Lutzomyia, in the New World. Approximately 700 species of phlebotomine sand flies have been described, of which about 
TABLE 1 Leishmania species that cause leishmaniasis in Brazil. ${ }^{3,4}$

Tegumentary leishmaniasis

Visceral leishmaniasis

\begin{tabular}{lllll}
\hline Localized cutaneous & Disseminated cutaneous & Mucosal & Diffuse cutaneous & \\
\hline L. $($ V. $)$ braziliensis & L. $($ V.) braziliensis & L. $($ V.) braziliensis & L. (L.) amazonensis & L. (L.) infantum (syn. chagasi) \\
\hline L. $($ V. $)$ guyanensis & L. $($ L. $)$ amazonensis & L. $($ L) amazonensis* & & \\
\hline L. $($ L. $)$ amazonensis & & L. (L.) guyanensis* & &
\end{tabular}

L. (V.) Iainsoni*

L. (V.) naiffi*

L. (V.) shawi*

L. (V.) lindenbergi*

*Less frequent species.

30 species are proven vectors of leishmaniasis and more than 40 additional species are suspected vectors. ${ }^{6}$

Transmission of the disease occurs through the infected insect's bite at the time of hematophagy, and nonvector transmission (for example, an accident in the research laboratory) is rare. However, in the case of VL, other possible routes of transmission have also been reported, such as congenital transmission, blood transfusion and syringe sharing among drug users. ${ }^{3}$

\section{Host-parasite interactions}

Protozoa of the genus Leishmania alternate between two main morphological forms during their life cycle: amastigotes and promastigotes, which are found in vertebrate hosts and phlebotomine vectors, respectively (Figure 1). ${ }^{7}$

The immobile amastigote forms are spherical or oval and measuring about 2.5-5.0 $\mu \mathrm{m}$ in diameter. They have a kinetoplast located close to the nucleus and must be multiplied within the cells of the vertebrate host's mononuclear phagocytic system. Thus, phlebotomine females become infected during blood feeding on a vertebrate host by ingesting blood and/or interstitial lymph containing macrophages parasitized by amastigote forms of Leishmania. ${ }^{8}$

In the insect's digestive tract, the amastigotes differentiate into promastigotes, which are elongated, flagellated, mobile forms around 5-15 $\mu \mathrm{m}$ in length and with kinetoplasts located between the nucleus and the anterior extremity. While still in the vector's digestive tract, the promastigote forms go through several stages - procyclical, nectomonate, leptomonate, haptomonate - until they become metacyclic promastigotes, which are infeccious to the vertebrate host. ${ }^{8}$

Highly adapted for successful transmission, the metacyclic promastigotes migrate to the insect's mouthparts (proboscis). Consequently, the vertebrate hosts are infected when female sand flies inoculate the metacyclic promastigote forms together with saliva during hematophagy. ${ }^{9}$
Once within the vertebrate host, the promastigotes are internalized by macrophages and, within the phagocytic vacuole, they are transformed into amastigotes, which replicate intensely until they rupture the parasitized cell. The released amastigotes infect other macrophages and the cycle starts again. ${ }^{10}$

Several adaptation mechanisms have been developed by parasites of the genus Leishmania in order to ensure their survival in the different hostile environments faced throughout their entire life cycle. These parasites not only deal with the aggressive digestive conditions found within phlebotomine sand flies, but must also prevent destruction by the immune system of the vertebrate host and ensure their survival within the macrophages. ${ }^{11}$

Regarding development in the vector, some of the adaptation strategies include: (1) expression of molecules on the cell surface such as LPG (lipophosphoglycan) and metalloprotease gp63, which protect the parasite from the hydrolytic enzymes present in the insect's intestine; (2) adherence of the nectomonate promastigote forms to the intestinal epithelial cells in order to avoid elimination with the vector's feces after digestion of the ingested blood; (3) structural modifications in the LPG molecules of the metacyclic promastigote forms, enabling their migration to the insect's mouthpiece; and (4) secretion of promastigote secretory gel (PSG) produced by the leptomonate forms, which favors transmission to the vertebrate host through regurgitation of the parasites. ${ }^{9,12}$

Transiently in the vertebrate host's bloodstream, before infecting the macrophages, the first immune system barrier encountered by the parasites after transmission is the complement system. Molecules present on the surface of the metacyclic promastigote forms, such as LPG and gp63, confer resistance to complement-mediated lysis. LPG prevents insertion of the C5b-9 complex into the membrane and gp 63 promotes $\mathrm{C} 3 \mathrm{~b}$ cleavage at $\mathrm{C} 3 \mathrm{bi}$ on the surface of the parasite, preventing the formation 
of $\mathrm{C} 5$ convertase. Thus, both molecules prevent the formation of the membrane attack complex. ${ }^{13}$

After escaping from the extracellular environment, the parasites penetrate the phagocytic cells through interactions between their surface molecules and the receptors of the macrophages. Metacyclic promastigotes not only resist complement-mediated lysis, but also use it in their favor for entry into macrophages. Indeed, the main internalization mechanism of leishmaniasis depends on the interaction of $\mathrm{C} 3 \mathrm{~b}$ and $\mathrm{C} 3 \mathrm{bi}$ molecules, which bind to the surface of the parasite with their respective receptors present in the macrophages, CR1 and CR3. ${ }^{11}$ The internalization of the parasites through CR3 receptors is an important escape mechanism, given that in this process the microbicidal respiratory explosion mechanism is not activated and IL-12 production induced by cell-mediated immunity is inhibited. ${ }^{13}$

Several other receptors present on macrophages are described as facilitators of the internalization of the promastigote forms of different species of Leishmania. For example, metacyclic promastigote forms can also be opsonized with IgG molecules and internalized by binding to $\mathrm{Fc}$ receptors. Furthermore, they can be phagocytosed by the interaction of the LPG molecules with mannosefucose receptors. ${ }^{11}$ In addition, LPG molecules may also interact with C-reactive protein (CRP), one of the first products of inflammatory response, and cause phagocytosis by means of CRP receptors. ${ }^{14}$ Furthermore, the gp63 and LPG molecules may also interact with fibronectin and CR4 receptors, respectively. ${ }^{15}$

After binding to the cell surface of the macrophages, the promastigote forms of the parasite are endocytosed in a phagosome, which after a series of fusion events becomes a phagolysosome. Unlike amastigote forms, promastigote forms are vulnerable to the acid and hydrolytic degradation of the phagolysosome. Thus, the first defense mechanism of the parasite inside the macrophages consists of the delay in the formation of the phagolysosome, with this process dependent on the LPG surface molecules, the presence of calcium and the inhibition of the protein kinase $\mathrm{C}(\mathrm{PKC}) .{ }^{16,17}$

Once the phagolysosome is formed, the promastigotes become amastigotes, which are more resistant to the microbicidal activity of the macrophages, since they can inhibit the hydrolytic enzymes, the production of nitric oxide and also the metabolites of the oxidative burst..$^{12}$ In addition, Leishmania have developed several strategies to escape the host's immunological defense, including: (1) inhibition of the host cell's ability to present antigens of the parasite to other components of the immune system, by means of interference in the expression of MHC class II molecules and lower expression of costimulatory molecules, such as B7-1 and CD40; (2) inhibition of the production of the cytokines involved in the proinflammatory response (IL-1, TNF- $\alpha$, IL-6) and activation of T lymphocytes (IL-12); and (3) induction of immunosuppressive molecules, such as prostaglandin E2 (PGE2), TGF- $\beta$ and IL-10. ${ }^{11,18}$

In recent decades, it has been found that even the saliva of vectors has biomolecules such as maxidilan which, in addition to facilitating the hematophagy process, also favor the transmission of the parasite. These biomolecules have immunomodulatory properties that interfere in the presentation of antigens by the macrophages and in the production of cytokines, increasing the production of IL- 4 and IL- 6 and inhibiting TNF- $\alpha$, IFN- $\gamma$ and IL-12. ${ }^{19}$

The intracellular location of the amastigote forms of Leishmania causes infection control to be dependent on the cell-mediated immune response, characterized by increased CD4+ T lymphocytes. As with other infectious and parasitic diseases, numerous studies in experimental models establish a "Th1/Th2 paradigm" in leishmaniasis, in which resistance to disease is conferred by a Th1 response with elevated levels of IFN- $\gamma$, IL-12, IL-2 and TNF- $\alpha$. This stimulates the microbicidal function of macrophages and promote the death of intracellular parasites - while susceptibility is linked to a Th2-type response, with an increase in the production of IL-4, IL-5, IL-10 and IL-13, inhibiting the activation of macrophages and contributing to parasite growth in the lesions..$^{20}$ However, resistance or susceptibility to disease in humans is not explained exclusively by the Th1 or Th2 response pattern, and the immune response may be strongly influenced by factors such as malnutrition, immune suppression (e.g. HIV) and, inevitably, the host's genetic components. ${ }^{21}$

At the same time that the Th1 response plays a clearly immunoprotective role, the high production of cytokines such as IFN- $\gamma$, TNF- $\alpha$ and IL-12 can be toxic and contribute to the pathogenesis of leishmaniasis. Thus, the Th1 response formed after infection is often accompanied by the response of CD $4+\mathrm{CD} 25+$ regulatory $\mathrm{T}$ cells (Treg) that produce IL-10 and TGF- $\beta$, which, in turn, block the excessive activation of Th1 cells and, consequently, prevent tissue damage. ${ }^{22}$

Recently, special attention has been given to the role of Th17 cells in the immune response against leishmaniasis. These cells differentiate from naive $\mathrm{CD} 4+\mathrm{T}$ lymphocytes in the presence of TGF- $\beta$ and IL- 6 , and secrete cytokines such as IL-21, IL-17 and IL-22, which operate in the inflammatory process. However, the specific role of these cells in leishmaniasis still remains inconclusive, given that the studies are controversial as to their contribution in the resistance or susceptibility to infection. ${ }^{23,24}$ 


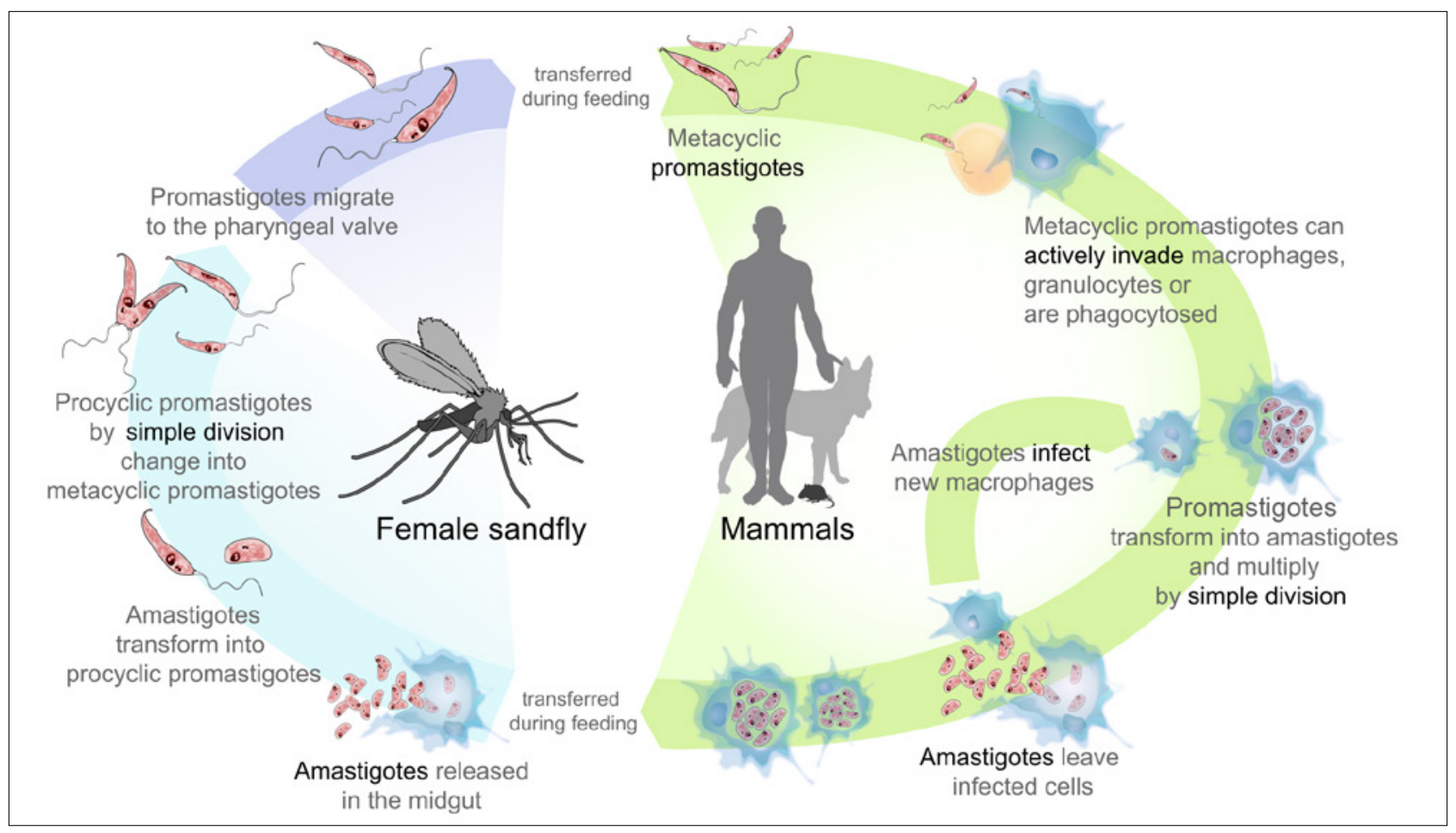

FIGURE 1 Life cycle of the parasites from the genus Leishmania, the cause of the disease leishmaniasis. Source: Wikimedia Commons (https://commons.wikimedia.org/wiki/File:Leishmaniasis_life_cycle_diagram_en.svg)

\section{Clinical, histopathological and IMMUNOLOGICAL ASPECTS}

In fact, the great variety of Leishmania responsible for CL and $\mathrm{VL}$ combined with the immune mechanisms of the host facilitates the existence of different clinical, histopathological and immunopathological manifestations. Considering the host's forms of response, the location of the lesions from the vector bite site and the clinical evolution, CL can be further classified as localized cutaneous leishmaniasis (LCL), disseminated cutaneous leishmaniasis (DL), diffuse cutaneous leishmaniasis (DCL) and mucosal leishmaniasis (ML) (Figure 2). ${ }^{4}$

LCL, the most frequent clinical manifestation, is characterized by the presence of an exclusively cutaneous lesion at the site of the phlebotomine sand fly bite, usually on exposed areas of the skin such as the face, hands and legs. The lesion begins with redness and swelling, increases in size progressively and, after a variable incubation period that usually lasts from 10 days to 3 months, develops into a typical ulcer with a rounded or oval shape and erythematous base. It is infiltrated and of firm consistency, well delimited and has elevated borders and a reddish background with coarse granulations. The lesion is usally painless; however, if there is an associated bacterial infec- tion, local pain and the production of seropurulent exudate may occur. ${ }^{25}$

In general, LCL ulcers have few parasites and the patient's cellular immunity is preserved, including a strong T-cell response, with a predominance of Th1-type cytokines (IFN- $\gamma$ and IL-12). ${ }^{26}$ If left untreated, depending on the species of the parasite and the host's immune response, the lesion tends to heal spontaneously over a period of a few months to a few years, and may also remain active for a long time. ${ }^{4}$

$\mathrm{DL}$, which is the disseminated form of CL, is a relatively rare expression that probably occurs due to hematogenous or lymphatic dissemination of the parasite. In these cases, the skin lesions are numerous, and are generally small and ulcerated, and distributed over several areas of the body. Parasites in the lesions are rare or absent and the immune response is quite varied. There appears to be incomplete inhibition of $\mathrm{T}$ cells, yet there is an evident supremacy of the Th1 response over Th2. ${ }^{27}$

ML is clinically expressed by destructive lesions located in the mucosa of the upper airways, possibly due to the spread of parasites to these areas, usually after LCL with chronic evolution, due to lack of treatment or inadequate treatment. In general, the initial clinical manifes- 
tations include nasal obstruction, hyperemia, formation and elimination of crusts by nasal mucosa and epistaxis. The nasal mucosa is involved in almost all cases, mainly affecting the cartilaginous septum, lateral walls, vestibule, and head of the inferior turbinate and, secondarily, the palate, lips, tongue, pharynx and larynx. There may be a progressive increase in the volume of the nose, destruction of the nasal septum cartilage with collapse of the tip of the nose, complete destruction of the nose and surrounding areas (with swallowing and speech disturbances), and significant mutilation of the face, leading to death due to complications from secondary infections. ${ }^{25}$

Immunologically, ML is characterized by a high specific cellular immune response, both Th1 and Th2. In these cases, there are high levels of proinflammatory cytokines (TNF- $\alpha$ and IFN- $\gamma$ ) and IL-4, and decreased levels of IL-10 and TGF- $\beta$, which explains the chronic and severe tissue destruction and the scarcity of parasites in the lesions. ${ }^{28}$ Untreated mucosal lesions are normally progressive and, even when treated, may leave behind sequelae such as nasal pyramid retraction, septum or palate perforation and destruction of the uvula, among others. ${ }^{4}$
DCL is a rare and severe clinical form of CL, which occurs in patients considered as being anergic, with deficiency in the cellular immune response to Leishmania antigens. Initially insidious, with a single lesion, it evolves in a chronic manner, with the formation of infiltrated plaques and multiple non-ulcerated nodulations that cover large cutaneous extensions. Generally, many parasites are found in the lesions and the cytokine profile of the patients is predominantly of the Th2-type, with low IFN- $\gamma$ production and high levels of IL-4 and IL-10. As a rule, the nodular lesions do not heal spontaneously and are resistant to available treatments. ${ }^{29}$

$\mathrm{VL}$, also known as kala-azar, is a chronic, systemic disease that mainly affects the lymph nodes, spleen, liver and bone marrow, and, less commonly, the kidneys, Peyer's patches in the intestine, the lungs and skin. The incubation period of the disease is quite variable, ranging from 10 days to 24 months (with an average between 2 to 6 months), and the main discrete or marked clinical manifestations include fever, hepatomegaly, splenomegaly, cutaneous/mucosal pallor, diarrhea and weight loss. Often, complementary tests show different degrees of anemia,

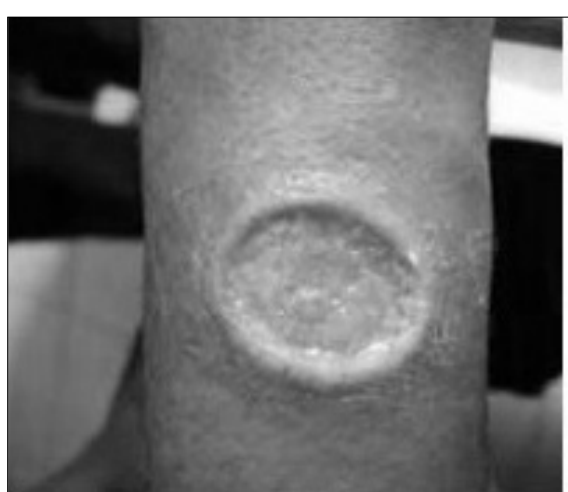

Localized cutaneous

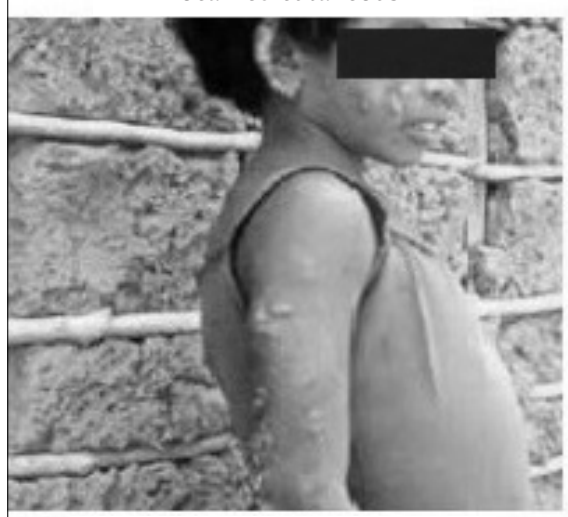

Diffuse cutaneous

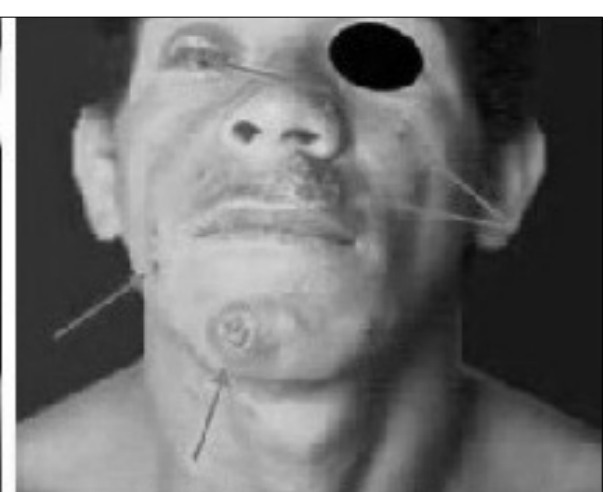

Disseminated cutaneous

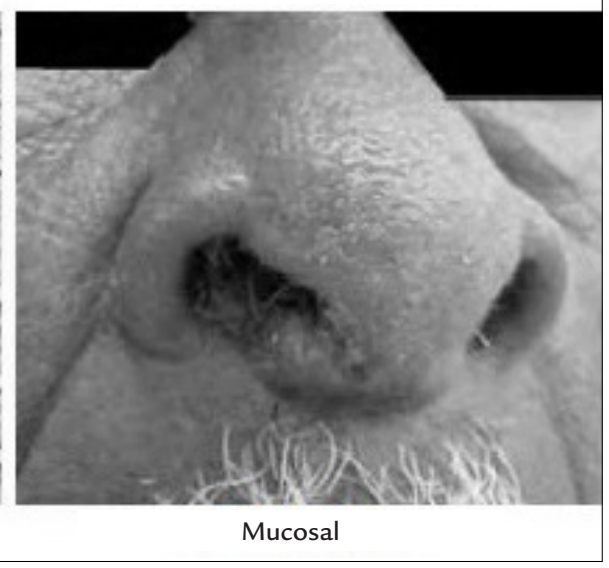

FIGURE 2 Clinical classifications of tegumentary leishmaniasis. Modified from: Ministério da Saúde. ${ }^{4}$ 
thrombocytopenia, and leukopenia with marked predominance of lymphomonocyte cells, hypoalbuminemia and hypergammaglobulinemia. ${ }^{5,30}$

As for the immunological aspects of $\mathrm{VL}$, these have not yet been clearly defined. In these cases, the Th1-resistance and Th2-susceptibility paradigm may be a banalization of a much more complex network of interactions, given that high levels of specific antibodies are also observed in this form of the disease..$^{31}$ Left untreated, VL almost always progresses to death and, even when treated, the disease can result in rates of fatal cases of around $10-20 \%$, with death often being caused by bacterial infections and/or bleeding. ${ }^{32}$

\section{Diagnosis}

The diagnosis of leishmaniasis involves the association of the clinical, epidemiological and laboratory aspects of the disease, and the application and sensitivity of each method may vary according to the clinical forms, the time of evolution of the lesions and the different species of Leishmania involved. ${ }^{3}$

The techniques that enable the demonstration of microscopic parasites constitute the "gold standard" in the diagnosis of the disease due to their specificity. In CL, investigation of the parasite can be done by scarification, biopsy, imprint and puncture aspiration, usually performed at the edge of the lesion. These are fast and inexpensive techniques, although they have limited sensitivity, especially in chronic lesions. In the case of VL, viewing of the parasites in tissue samples requires invasive procedures, and therefore has limited indications. Bone marrow aspirate is the most used method and its sensitivity is between $60 \%$ and $85 \%$. Splenic aspiration has a sensitivity level higher than $95 \%$, but is usually not conducted due to the risk of bleeding. Although less risky, liver and lymph node punctures show very low sensitivities of approximately $45 \%$. In vitro cultivation and the inoculum of the material obtained from clinical samples in animals may improve the positivity of the result and the safety of the diagnosis. However, these methods are rarely used in clinical practice, since, in addition to the complexity and high cost, the parasites' growth can take weeks or months. ${ }^{33}$

Polymerase chain reaction (PCR) has shown promising results in the diagnosis of leishmaniasis. In addition to presenting high sensitivity, PCR can be performed from different clinical samples, including peripheral blood in cases of VL, and enables the characterization of the Leishmania species involved, depending on the technique employed. Despite being widely used for research purposes, it is not used often in the diagnostic routine, as in addition to the high cost the technique requires standardization, laboratory infrastructure and technical rigor. ${ }^{29}$

Serological techniques - such as indirect immunofluorescence (IFAT), direct agglutination (DAT) and enzyme-linked immunosorbent assay (ELISA) -, which are standardized for the detection of anti-Leishmania antibodies, are important tools for the diagnosis of cases of VL. These are usually associated with a prominent humoral response. However, they are not commonly used in cases of CL, due to variable sensitivity and specificity rates and reduced levels of antibodies, especially in cases of LCL. ${ }^{33}$ As such, immunochromatographic assays have been evaluated in different endemic regions aimed at diagnosing $\mathrm{VL}$ in the field. In Brazil, the test used the most and recommended by the Ministry of Health is the rapid test with the recombinant antigen $\mathrm{k} 39$, which has shown sensitivity ranging from $86-100 \%$ and specificity of $82-100 \%{ }^{34}$ It is worth noting that serological tests also present important limitations: (a) individuals in endemic areas with positive serology, without signs and symptoms, may or may not develop the disease; (b) positive serology is not necessarily related to active disease, as elevated levels of antibodies may remain in the patient's serum for a long period after clinical cure; (c) in cases of HIV coinfection, serological tests are often negative, due to the lower level of circulating antibodies resulting from immunosuppression. ${ }^{35}$

In general, a differential diagnosis should always be considered. In CL cases, numerous skin lesions resulting from other diseases may mimic clinical and epidemiological aspects common to leishmaniasis, such as syphilis, leprosy, tuberculosis, paracoccidioidomycosis, histoplasmosis, chromoblastomycosis, sporotrichosis, pyoderma, discoid lupus erythematosus, psoriasis, Jessner lymphocytic infiltrate, vasculitis and cutaneous neoplasias, among others. ${ }^{4}$ As for VL, diseases that also cause febrile hepatosplenomegaly, such as malaria, brucellosis, typhoid fever, schistosomiasis and the acute form of Chagas disease are also prominent, as well as hematological disorders such as lymphoma, multiple myeloma and sickle cell anemia. ${ }^{5}$

\section{TrEatMent}

Successful treatment involves several factors, such as: (1) host factors such as genetics, immune response and clinical presentation of the disease; (2) treatment resources, such as quality of the drug, dosage, and duration and completion of the therapy; and (3) characteristics of the parasite, such as intrinsic sensitivity of the species and lack of resistance to the medication. ${ }^{36}$

The drugs of first choice in the treatment of all clinical forms of leishmaniasis are pentavalent antimonials 
$(\mathrm{Sb}+5)$, sodium stibogluconate $\left(\right.$ Pentostan $\left.^{\circledR}\right)$ and N-methylglucamine antimoniate (Glucantime ${ }^{\circledR}$ ), with the latter marketed and distributed solely by the Ministry of Health in Brazil. ${ }^{4,5}$ Both are administered parenterally and interfere with the bioenergetics of the amastigote forms of the parasite, inhibiting the glycolytic activity and oxidative pathway of fatty acids, with consequent reductions in ATP production and molecular biosynthesis. ${ }^{37}$

Despite pentavalent antimonials showing an effectiveness of approximately $90 \%$ in most studies (with the exception of cases of DCL), these drugs have toxic side effects on the cardiac, kidney and liver systems. ${ }^{38}$ Therefore, they are contraindicated for patients suffering from heart, kidney and liver diseases, and also for pregnant women, as they are able to cross the transplacental barrier and affect the fetal nervous tissue, leading to severe mental retardation syndromes., ${ }^{4,5}$

In recent years, the emergence of resistance to pentavalent antimonials in the parasite has limited treatment in various countries. In some parts of India, for example, failure rates of more than $60 \%$ have been observed in the treatment of VL caused by L. (L.) donovani. ${ }^{36}$ In Latin America, the rates also vary, even in LCL cases. ${ }^{39}$ One of the factors that has certainly contributed to increased resistance is the generalized misuse of the drug (such as insufficient dosages, irregular and incomplete therapies). ${ }^{40}$

If there is no satisfactory response to therapy with pentavalent antimonials or if it is not possible for them to be used, the drugs of second choice in the treatment of leishmaniasis are amphotericin B and pentamidine. ${ }^{4,5}$

Amphotericin B deoxycholate (Fungizone) is an antibiotic normally used for the treatment of systemic fungal infections. It has good activity in the destruction of leishmaniasis, with cure rates of over $97 \%$ for both CL and VL (depending on the species involved). ${ }^{38}$ Said drug acts selectively on the amastigotes and promastigotes forms of the parasite, through preferential attachment with esters present in the plasma membrane. In addition, it causes an increase in the synthesis of nitric oxide by the macrophages ${ }^{41}$ However, treatment with amphotericin $B$ is quite arduous and can only be performed in a hospital environment (intravenous). Adverse effects are numerous and frequent, including fever, headache, nausea, phlebitis, cyanosis, anemia, leukopenia, hypotension, hypokalemia, hypomagnesemia, cardiovascular alterations and renal complications. ${ }^{38}$ In 1997, a new formulation of amphotericin B became commercially available - liposomal amphotericin B (AmBisome). This new drug, which is also administered intravenously, achieves high concentrations in the liver and the spleen and low concentrations in the lungs and kidneys. Therefore, it is less toxic and induces fewer side effects. ${ }^{42}$ Thus, it is recommended for patients with VL and renal and/or cardiac complications, and also for patients considered vulnerable, such as pregnant women and HIV-positive people. ${ }^{5}$ However, there are limitations that restrict its use, including its high cost, efficiency variations between regions, slow intravenous administration, thermal instability and adverse reactions related to the infusion. ${ }^{42}$ Additionally, in the treatment of CL, there are still no controlled clinical trials that support the use of liposomal amphotericin ${ }^{4}$ and the few isolated studies that exist demonstrate varied cure rates. ${ }^{43}$

Pentamidine comes in the form of two salts: mesylate and isethionate (available in Brazil). Such drugs are used primarily in regions where the failure of treatment with pentavalent antimonials is common - especially in India, French Guiana and Suriname - and in individual cases of resistance to the first therapy of choice. ${ }^{38}$ The main limitations involving the use of pentamidine are related to its side effects such as headache, nausea, abdominal pain, hypoglycemia, tachycardia, kidney failure in $25 \%$ of patients (usually reversible) and pancreatitis that can lead to the onset of diabetes mellitus, in 10 to $15 \%$ of cases. Therefore, pentamidine is contraindicated in cases of pregnancy, diabetes mellitus, kidney failure, liver failure, heart disease and for children weighing less than $8 \mathrm{~kg} ., 4$ Another factor that has made the use of these drugs unfeasible is the emergence of parasitic resistance, meaning that efficacy has decreased over the years and cure rates below $70 \%$ have been reported. ${ }^{40}$

In recent decades, many researchers have focused their studies on the search for alternative treatments. Paromomycin (aminosidine), an aminoglycoside antibiotic administered parenterally, began to be used in the treatment of VL initially in India. ${ }^{44}$ Due to its low cost and infrequent side effects, which may include nephrotoxicity and ototoxicity, paromomycin represents an alternative for cases of resistance to the drugs of the first choice. ${ }^{38}$ However, wide inter- and intra-regional efficacy variations are observed in the treatment of VL with paromomycin ${ }^{45}$ and the cure rates reported in CL treatment are often lower than those observed with the use of pentavalent antimonials. ${ }^{46}$

In 2002, the first oral drug in the treatment of VL miltefosine - was introduced in India. Despite the frequent gastrointestinal side effects, such as vomiting and diarrhea, and possible transient elevations of hepatic transaminases, miltefosine represents a major advance in the treatment of the disease because it appears to be well tolerated and exhibits cure rates above $90 \%$ in cases of VL. ${ }^{47}$ However, in recent years, the relatively high cost, concerns about tera- 
togenicity and the potent development of resistance have limited the use of miltefosine in several countries. ${ }^{48}$

Currently, high expectations have now been raised about drug combination therapy in order to reduce the dosage and duration of treatment and thereby improve the tolerance and conformity of the medication already available on the market. ${ }^{36}$ Therefore, the search for new therapies or alternatives against the different forms of leishmaniasis remains a clinical priority.

\section{Conclusion}

Despite advances in scientific knowledge, leishmaniasis remains a major public health problem in several countries, with the disease spreading to areas that were previously nonendemic. Many challenges still have to be overcome in combating this pathology, emphasizing actions that are focused on early diagnosis, the formulation of new drugs and therapeutic regimes, as well as flexible, distinct and adequate control strategies for each transmission pattern, considering local environmental, social and economic characteristics.

\section{Resumo}

Leishmaniose humana no Brasil: uma revisão geral

A leishmaniose representa um complexo de doenças com amplo espectro clínico e diversidade epidemiológica, sendo considerada um grande problema de saúde pública. O presente artigo apresenta uma revisão geral sobre os ciclos de transmissão, as interações parasito-hospedeiro, os aspectos clínicos, histopatológicos e imunológicos, o diagnóstico e o tratamento das diversas formas da doença humana.

Palavras-chave: Leishmaniose. Infecções por Protozoários. Revisão.

\section{References}

1. Lainson R, Shaw J. Evolution, classification and geographical distribution. In: Peters W, Killick-Kendrick R, eds. The leishmaniasis in Biology and Medicine, Biology and Epidemiology. London: Academic Press; 1987. p.1120.

2. Alvar J, Vélez ID, Bern C, Herrero M, Desjeux P, Cano J, et al. Leishmaniasis worldwide and global estimates of its incidence. PLoS One. 2012;7(5):e35671.

3. Piscopo TV, Mallia Azzopardi C. Leishmaniasis. Postgrad Med J. 2007;83(976):649-57.

4. Ministério da Saúde. Secretaria de Vigilância em Saúde. Manual de vigilância da leishmaniose tegumentar. Brasília: Ministério da Saúde; 2017.

5. Ministério da Saúde. Secretaria de Vigilância em Saúde. Manual de vigilância e controle da leishmaniose visceral. Brasília: Ministério da Saúde; 2014.

6. Akhoundi M, Kuhls K, Cannet A, Votýpka J, Marty P, Delaunay P, et al. A Historical overview of the classification, evolution, and dispersion of leishmania parasites and sandflies. PLoS Negl Trop Dis. 2016;10(3):e0004349.
7. Hoare CA, Wallace FC. Developmental stages of trypanosomatid flagellates: a new terminology. Nature. 1966;212:1358-996.

8. Bates PA, Rogers ME. New insights into the developmental biology and transmission mechanisms of Leishmania. Curr Mol Med. 2004;4(6):601-9.

9. Bates PA. Transmission of Leishmania metacyclic promastigotes by phlebotomine sand flies. Int J Parasitol. 2007;37(10):1097-106.

10. De Menezes JP, Saraiva EM, Rocha-Azevedo B. The site of the bite: Leishmania interaction with macrophages, neutrophils and the extracellular matrix in the dermis. Parasit Vectors. 2016;9:264.

11. Cunningham AC. Parasitic adaptive mechanisms in infection by Leishmania. Exp Mol Pathol. 2002;72(2):132-41

12. Alexander J, Satoskar AR, Russell DG. Leishmania species: models of intracellular parasitism. J Cell Sci. 1999;112(Pt 18):2993-3002.

13. Dos-Santos AL, Carvalho-Kelly LF, Dick CF, Meyer-Fernandes JR. Innate immunomodulation to trypanosomatid parasite infections. Exp Parasitol. 2016;167:67-75.

14. Culley FJ, Harris RA, Kaye PM, McAdam KP, Raynes JG. C-reactive protein binds to a novel ligand on Leishmania donovani and increases uptake into human macrophages. J Immunol. 1996;156(12):4691-6.

15. Brittingham A, Chen G, McGwire BS, Chang KP, Mosser DM. Interaction of Leishmania gp63 with cellular receptors for fibronectin. Infect Immun. 1999;67(9):4477-84.

16. Tejle K, Magnusson KE, Rasmusson B. Phagocytosis and phagosome maturation are regulated by calcium in $\mathrm{J774}$ macrophages interacting with unopsonized prey. Biosci Rep. 2002;22(5-6):529-40.

17. Holm A, Tejle K, Gunnarsson T, Magnusson KE, Descoteaux A, Rasmusson B. Role of protein kinase $\mathrm{C}$ alpha for uptake of unopsonized prey and phagosomal maturation in macrophages. Biochem Biophys Res Commun. 2003;302(4):653-8.

18. Kima PE. The amastigote forms of Leishmania are experts at exploiting host cell processes to establish infection and persist. Int J Parasitol. 2007;37(10):1087-96.

19. Wheat WH, Pauken KE, Morris RV, Titus RG. Lutzomyia longipalpis salivary peptide maxadilan alters murine dendritic cell expression of CD80/86, CCR7, and cytokine secretion and reprograms dendritic cell-mediated cytokine release from cultures containing allogeneic $\mathrm{T}$ cells. J Immunol. 2008;180(12):8286-98.

20. Sharma U, Singh S. Immunobiology of leishmaniasis. Indian J Exp Biol. 2009;47(6):412-23.

21. Handman E, Elso C, Foote S. Genes and susceptibility to leishmaniasis. Adv Parasitol. 2005;59:1-75.

22. Campanelli AP, Roselino AM, Cavassani KA, Pereira MS, Mortara RA, Brodskyn $\mathrm{CI}$ et al. CD4+CD25+ T cells in skin lesions of patients with cutaneous leishmaniasis exhibit phenotypic and functional characteristics of regulatory T cells. J Infect Dis. 2006;193(9):1313-22.

23. Nascimento MS, Carregaro V, Lima-Júnior DS, Costa DL, Ryffel B, Duthie MS, et al. Interleukin $17 \mathrm{~A}$ acts synergistically with interferon $\gamma$ to promote protection against Leishmania infantum infection. J Infect Dis. 2015;211(6):1015-26.

24. Terrazas C, Varikuti S, Kimble J, Moretti E, Boyaka PN, Satoskar AR. IL-17A promotes susceptibility during experimental visceral leishmaniasis caused by Leishmania donovani. FASEB J. 2016;30(3):1135-43.

25. Handler MZ, Patel PA, Kapila R, Al-Qubati Y, Schwartz RA. Cutaneous and mucocutaneous leishmaniasis: clinical perspectives. J Am Acad Dermatol. 2015;73(6):897-908.

26. Scott $\mathrm{P}$, Novais FO. Cutaneous leishmaniasis: immune responses in protection and pathogenesis. Nat Rev Immunol. 2016;16(9):581-92.

27. Silveira FT, Lainson R, Castro Gomes CM, Laurenti MD, Corbett CE. Immunopathogenic competences of Leishmania. (V.) braziliensis and L. (L.) amazonensis in American cutaneous leishmaniasis. Parasite Immunol. 2009;31(8):423-31.

28. Amato VS, Andrade HF, Duarte MI. Mucosal leishmaniasis: in situ characterization of the host inflammatory response, before and after treatment. Acta Trop. 2003;85(1):39-49.

29. Kevric I, Cappel MA, Keeling JH. New world and old world leishmania infections: a practical review. Dermatol Clin. 2015;33(3):579-93.

30. Kurşun E, Turunç T, Demiroğlu YZ, Solmaz S, Arslan H. Evaluation of fourteen adult cases with visceral leishmaniasis. Mikrobiyol Bul. 2013;47(3):500-6.

31. Bhattacharya P, Ali N. Involvement and interactions of different immune cells and their cytokines in human visceral leishmaniasis. Rev Soc Bras Med Trop. 2013;46(2):128-34. 
32. Bern C, Maguire JH, Alvar J. Complexities of assessing the disease burden attributable to leishmaniasis. PLoS Negl Trop Dis. 2008;2(10):e313.

33. Von Stebut E. Leishmaniasis. J Dtsch Dermatol Ges. 2015;13(3):191-200.

34. Assis TS, Braga AS, Pedras MJ, Oliveira E, Barral A, Siqueira IC, et al. Multicentric prospective evaluation of $\mathrm{rk} 39$ rapid test and direct agglutination test for the diagnosis of visceral leishmaniasis in Brazil. Trans R Soc Trop Med Hyg. 2011;105(2):81-5.

35. Ejazi SA, Ali N. Developments in diagnosis and treatment of visceral leishmaniasis during the last decade and future prospects. Expert Rev Anti Infect Ther. 2013;11(1):79-98.

36. Sundar S, Singh A. Recent developments and future prospects in the treatment of visceral leishmaniasis. Ther Adv Infect Dis. 2016;3(3-4): 98-109.

37. Wyllie S, Cunningham ML, Fairlamb AH. Dual action of antimonial drugs on thiol redox metabolism in the human pathogen Leishmania donovani. J Biol Chem. 2004;279(38):39925-32.

38. Croft SL, Yardley V. Chemotherapy of leishmaniasis. Curr Pharm Des. 2002;8(4):319-42

39. Tuon FF, Amato VS, Graf ME, Siqueira AM, Nicodemo AC, Amato Neto V. Treatment of New World cutaneous leishmaniasis: a systematic review with a meta-analysis. Int J Dermatol. 2008;47(2):109-24.

40. Croft SL, Sundar S, Fairlamb AH. Drug resistance in leishmaniasis. Clin Microbiol Rev. 2006;19(1):111-26.

41. Trajkovic V, Markovic M, Samardzic T, Miljkovic DJ, Popadic D, Mostarica Stojkovic M. Amphotericin B potentiates the activation of inducible nitric oxide synthase and causes nitric oxide-dependent mitochondrial dysfunction in cytokine-treated rodent astrocytes. Glia. 2001;35(3):180-8.

42. Balasegaram M, Ritmeijer K, Lima MA, Burza S, Ortiz Genovese G, Milani B, et al. Liposomal amphotericin B as a treatment for human leishmaniasis. Expert Opin Emerg Drugs. 2012;17(4):493-510

43. Wortmann G, Zapor M, Ressner R, Fraser S, Hartzell J, Pierson J, et al Liposomal amphotericin B for treatment of cutaneous leishmaniasis. Am J Trop Med Hyg. 2010;83(5):1028-33.

44. Thakur CP, Kanyok TP, Pandey AK, Sinha GP, Messick C, Olliaro P. Treatment of visceral leishmaniasis with injectable paromomycin (aminosidine) An open-label randomized phase-II clinical study. Trans R Soc Trop Med Hyg. 2000;94(4):432-3.

45. Hailu A, Musa A, Wasunna M, Balasegaram M, Yifru S, Mengistu G, et al Geographical variation in the response of visceral leishmaniasis to paromomycin in East Africa: a multicentre, open-label, randomized trial. PLoS Negl Trop Dis. 2010;4(10):e709.

46. Hepburn NC, Tidman MJ, Hunter JA. Aminosidine (paromomycin) versus sodium stibogluconate for the treatment of American cutaneous leishmaniasis. Trans R Soc Trop Med Hyg. 1994;88(6):700-3.

47. Bhattacharya SK, Jha TK, Sundar S, Thakur CP, Engel J, Sindermann H, et al. Efficacy and tolerability of miltefosine for childhood visceral leishmaniasis in India. Clin Infect Dis. 2004;38(2):217-21.

48. Sundar S, Singh A, Rai M, Prajapati VK, Singh AK, Ostyn B, et al. Efficacy of miltifosine in the treatment of visceral leishmaniasis in India after a decade of use. Clin Infect Dis. 2012;55(4):543-50. 